Received: 28 March 2018

Accepted: 5 July 2018

Published online: 01 August 2018

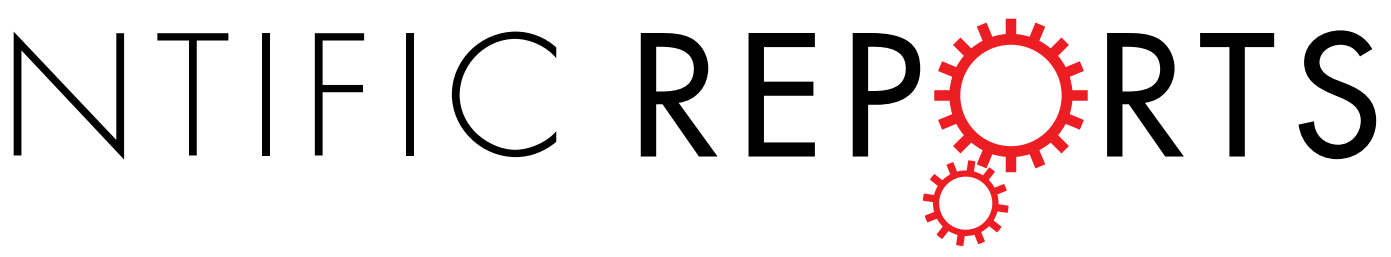

\title{
Serum uric acid predicts incident metabolic syndrome in the elderly in an analysis of the Brisighella Heart Study
}

Arrigo Francesco Giuseppe Cicero $\mathbb{D}$, Federica Fogacci $(\mathbb{D}$, Marina Giovannini, Elisa Grandi, Martina Rosticci, Sergio D’Addato \& Claudio Borghi

Several epidemiological studies report a positive correlation between hyperuricemia and metabolic syndrome (MetS) in adults, which hyperuricemic subjects seem to more easily develop. We aimed to verify if serum uric acid (SUA) concentrations were positively associated with MetS prevalence and middle-term (4-year) incidence in older overall healthy subjects. We also purposed to identify which SUA cut-off values could be functional in MetS diagnosis in addition to the traditionally used parameters. For this reason, we selected from the historical cohort of the Brisighella Heart Study 923 older healthy subjects repeatedly visited during the 2008 and 2012 population surveys. In our sample, MetS was more frequent for higher SUA concentrations rather than the population's mean in both men $[O R=2.12,95 \%$ C.I. $(1.55,2.90)]$ and women $[O R=2.69,95 \%$ C.I. $(1.91,3.78)] . R O C$ analysis showed SUA was predictive of MetS in the whole population $[A U C=0.647,95 \% C . I .(0.609,0.686), P=0.000001]$ and in both sex subgroups [men: $A U C=0.592,95 \% C .1 .(0.529,654) ; P=0.004$; women: $A U C=0.758$, 95\%C.I. $(0.711,0.806), P<0.000001]$, even there were sex-related differences in the best cut-off values $(5.5 \mathrm{mg} / \mathrm{dL}$ for men; $4.2 \mathrm{mg} / \mathrm{dL}$ for women). Prospectively, SUA appeared predictive of middle-term (4-year) MetS incidence in the whole population (AUC $=0.604,95 \% C .1 .[0.518,0.690], P=0.029$, best cut-off value $=4.7 \mathrm{mg} / \mathrm{dL}$ ) and in the female group $(A \cup C=0,641,95 \% C . I .[0.519,0.762], P=0.039$, best cut-off value $=3.9 \mathrm{mg} / \mathrm{dL}$ ) though not in the male one $(P>0.05)$. In conclusion, in our cohort, SUA is a frequent component of MetS, other than a middle-term predictor of newly diagnosed MetS in older women.

Currently evidence shows that subjects with high serum uric acid (SUA) levels have increased cardiovascular (CV) morbidity and mortality rates rather than the normouricemic ones ${ }^{1}$. However, it is controversial whether hyperuricemia is an independent risk factor for cardiovascular diseases (CVDs) developing or only a confounding one $e^{2}$, since many confirmed CVDs risk factors are per se associated with increased SUA ${ }^{3-6}$. Lacking a definitively proven causal association, a relationship of reverse causality has been also taken into account, whereby preclinical atherosclerosis could lead to higher levels of uric acid before a diagnosis of ischaemic heart disease ${ }^{7}$. There is also a growing evidence that SUA is involved in metabolic syndrome (MetS) incidence: several epidemiological studies showed a positive correlation between hyperuricemia and Met $S^{8}$, which hyperuricemic subjects seem to more easily develop 9 . In fact, even though the exact biological mechanism is not yet known, uric acid seems to irreversibly react with nitric oxide (NO), disabling it and leading to endothelial dysfunction and, consequently, promoting the development of hypertension and $\mathrm{Met}^{10}$. At the same time, NO has a well-known role in the insulin resistance and its shortage reduces the blood flow to the tissues sensitive to insulin, resulting in blocking of the insulin action ${ }^{11,12}$.

The aim of our study was at verifying if in a sample of overall healthy older subjects there is a correlation between SUA levels and MetS prevalence and middle-term (4-year) incidence. Moreover, we tried to identify SUA cut-off values, which could be functional as a diagnosis parameter of MetS in addition to the traditional ones.

Hypertension and Atherosclerosis Research Group, Medical and Surgical Sciences Department, Sant'Orsola-Malpighi University Hospital, Via Albertoni 15, 40138, Bologna, Italy. Correspondence and requests for materials should be addressed to A.F.G.C. (email: arrigo.cicero@unibo.it) 


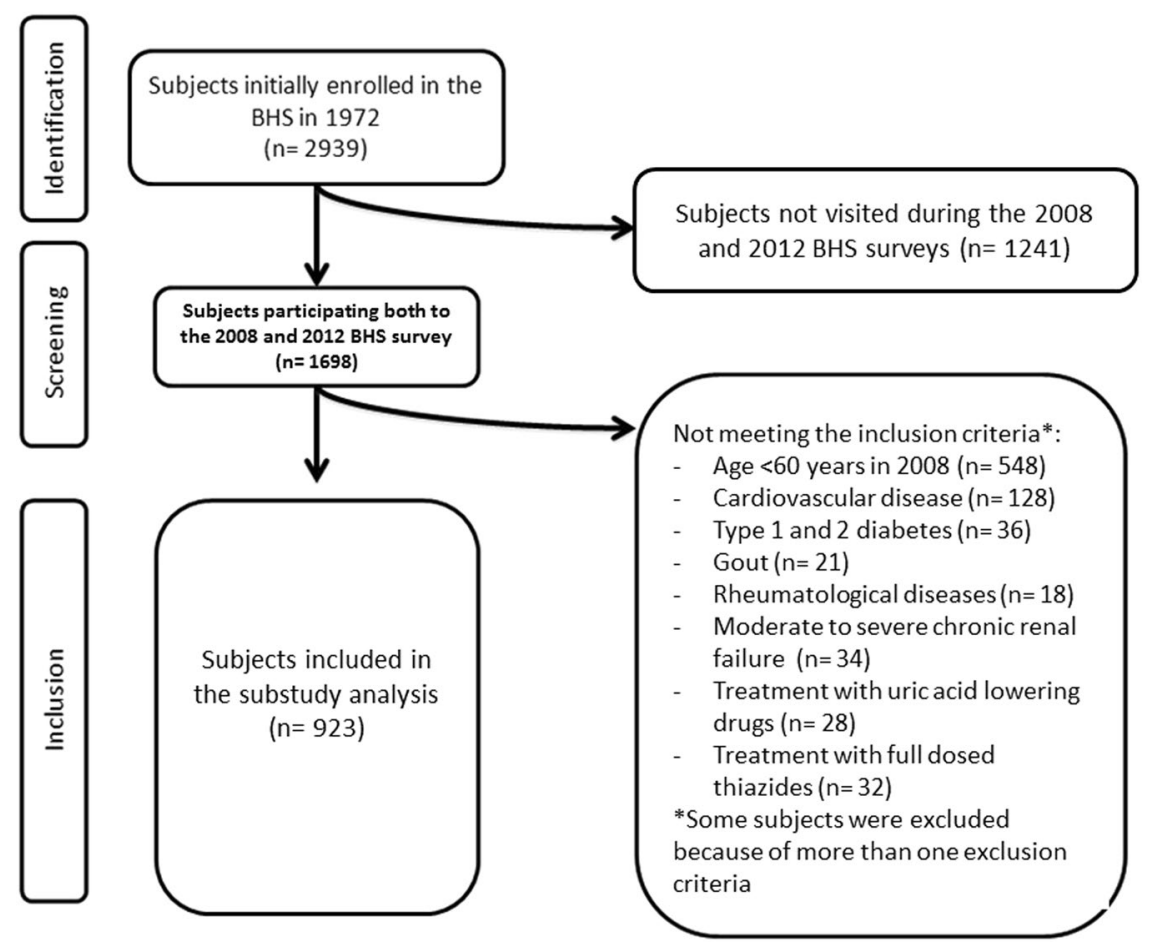

Figure 1. Flow-chart resuming the selection criteria applied to the full cohort to select the investigated subjects.

\section{Material and Methods}

The Brisighella Heart Study (BHS) is a prospective, longitudinal population-based investigation involving 2939 randomly selected Caucasian subjects resident in the northern Italian rural town of Brisighella. The BHS cohort consists in 1491 men and 1448 women, at enrolment aged 14 to 84 years and free from cardiovascular disease. The study started in 1972 and is still ongoing. The town of Brisighella was originally selected as the site for the study because of the homogeneity of life-style among its residents, with a very low rate of migration. Subjects were clinically evaluated at baseline and every four years thereafter, by collecting an extensive amount of clinical and laboratory data.

The BHS protocol and its sub-studies, which are largely described elsewhere ${ }^{13}$, have been approved by the Ethical Board of the University of Bologna and all of the involved volunteers gave their signed informed consent to participate ${ }^{14}$. All methods were performed in accordance with the relevant guidelines and regulations.

Blood pressure (BP) measurements have been taken early in the morning, after a 10 minutes rest in a quiet room, while subjects were in the seated position and by the use of a validated oscillometric device, with a cuff of the appropriate size applied to the right upper arm. The mean value of three blood pressure readings (obtained at 1 -minute intervals) was considered as the variable of the study.

Laboratory analyses were carried out by trained personnel according to standardized methods ${ }^{15}$. They included total cholesterol (TC), triglycerides (TG), high-density lipoprotein cholesterol (HDL-C), low-density lipoprotein cholesterol (LDL-C), lipoprotein(a) (Lp(a)), apolipoprotein A-1 (Apo A-1), apolipoprotein B-100 (Apo B-100), fasting plasma glucose (FPG), serum uric acid (SUA), creatinine phosphokinase (CPK), estimated glomerular filtration rate (eGFR), total bilirubin (TB), aspartate aminotransferase (ALT), alanine aminotransferase (AST) and gamma-glutamyl-transferase (GGT).

For the purpose of the present study, we selected 923 older volunteers (age $>60$ years; 434 males and 489 females) repeatedly visited during the 2008 and the 2012 population surveys. Subjects pharmacologically treated with SUA increasing drugs (namely high dosed thiazide diuretics) and SUA lowering drugs, affected by cardiovascular disease, type 1 and 2 diabetes, gout and other rheumatological diseases, and/or moderate to severe chronic renal failure were excluded from the present analysis (Fig. 1).

MetS was identified based on the ATPIII/IDF criteria ${ }^{16}$. The components were defined using the following ATPIII categorizations: 1 ) high blood pressure (BP $\geq 130 / 85 \mathrm{mmHg}$ ); 2) hypertriglyceridemia (TG $\geq 150 \mathrm{mg} / \mathrm{dl}$ ), 3) low HDL-C ( $<40 \mathrm{mg} / \mathrm{dl}$ for men and $<50 \mathrm{mg} / \mathrm{dl}$ for women); 4) hyperglycaemia $(100 \mathrm{mg} / \mathrm{dl}>\mathrm{FPG})$, 5$)$ waist circumference $>102 \mathrm{~cm}$ for men and $>88 \mathrm{~cm}$ for women (suggestive of high abdominal obesity). Subjects with at least three of the previous components were classified as having MetS.

We carried out a full descriptive analysis of all the considered variables. Descriptive values were always expressed as mean \pm standard deviation (SD), because normally-distributedat the Kolmogorov-Smirnov normality test. Continuous parameters were compared by T-test for independent samples. First, we carried out a bivariate (Pearson) correlation for age, BMI, WC, SBP, DBP, TC, TG, HDL-C, LDL-C, Apo A-1, Apo B-100, AST, ALT, SUA, FPG and eGFR in overall population. Then, the analysis was repeated by sex. The risk estimate for MetS development was calculated and expressed as odds ratio (OR) and 95\% confidence interval (95\%C.I.). The 


\begin{tabular}{|l|c|c|c|}
\hline Parameters & $\begin{array}{l}\text { Whole population } \\
\mathbf{n = 9 2 3}\end{array}$ & Male $\mathbf{n = 4 3 4}$ & Female $\mathbf{n = 4 8 9}$ \\
\hline Age (years) & $72.0 \pm 5.6$ & $72.2 \pm 5.6$ & $71.8 \pm 15,6$ \\
\hline Body mass index $\left(\mathrm{Kg} / \mathrm{m}^{2}\right)$ & $26.1 \pm 4.2$ & $26.5 \pm 3.4$ & $25.6 \pm 4.7^{*}$ \\
\hline Waist Circumference $(\mathrm{cm})$ & $89.2 \pm 12.1$ & $93.2 \pm 10.6$ & $85.6 \pm 12.3$ \\
\hline Systolic blood pressure $(\mathrm{mmHg})$ & $131.6 \pm 16.6$ & $134.0 \pm 15.4$ & $129.4 \pm 17.4^{\S}$ \\
\hline Diastolic blood pressure $(\mathrm{mmHg})$ & $83.2 \pm 10.4$ & $85.6 \pm 10.0$ & $81.1 \pm 10.5^{\S}$ \\
\hline Total cholesterol (mg/dL) & $205.1 \pm 37.7$ & $205.1 \pm 38.2$ & $205.1 \pm 37.3$ \\
\hline Triglycerides $(\mathrm{mg} / \mathrm{dL})$ & $107.8 \pm 66.7$ & $116.1 \pm 71.7$ & $100.3 \pm 61.0^{\S}$ \\
\hline High-density lipoprotein cholesterol (mg/dL) & $46.5 \pm 9.7$ & $43.8 \pm 8.9$ & $49.0 \pm 9.8^{\S}$ \\
\hline Low-density lipoprotein cholesterol $(\mathrm{mg} / \mathrm{dL})$ & $137.3 \pm 33.2$ & $138.5 \pm 33.9$ & $136.2 \pm 32.5$ \\
\hline Apolipoprotein A1 (mg/dL) & $144.0 \pm 27.3$ & $135.0 \pm 25.3$ & $152.1 \pm 26.5^{\S}$ \\
\hline Apolipoprotein B (mg/dL) & $89.2 \pm 20.7$ & $92.0 \pm 21.0$ & $86.6 \pm 20.1^{\S}$ \\
\hline Fasting plasma glucose (mg/dL) & $100.8 \pm 9.6$ & $103.7 \pm 9.2$ & $98.1 \pm 9.2^{\S}$ \\
\hline Serum uric acid (mg/dL) & $4.8 \pm 1.4$ & $5.6 \pm 1.3$ & $4.1 \pm 1.1^{\S}$ \\
\hline Aspartate aminotransferase (U/L) & $23.3 \pm 9.1$ & $24.8 \pm 8.2$ & $21.9 \pm 9.7$ \\
\hline Alanine aminotransferase (U/L) & $24.3 \pm 16.1$ & $28.8 \pm 18.8$ & $20.3 \pm 11.9$ \\
\hline eGFR (mL/min/1.73 m $\left.{ }^{2}\right)$ & $84.2 \pm 5.8$ & $85.2 \pm 4.8$ & $83.4 \pm 6.5$ \\
\hline
\end{tabular}

Table 1. Main characteristics of the sample (2008), overall and sex-distributed, expressed as mean \pm standard deviation. eGFR, estimated glomerular filtration rate. ${ }^{*} \mathrm{P}=0.001 \mathrm{vs} \mathrm{men} ;{ }^{\mathfrak{s}} \mathrm{P}<0.001 \mathrm{vs}$ man.

receiver operating characteristic (ROC) curve analysis was firstly carried out using the MetS incidence at 2008 as state variable and the 2008's SUA as test variable, in overall population and by sex. Secondly, in order to assess the predictive value of SUA on the 4-year MetS incidence, the previous analysis was repeated considering only those subjects who were not affected by MetS in $2008(n=669)$ but though they became in 2012. ROC curve was drawn using the MetS incidence in 2012 as state variable and SUA (as assessed in the 2008) as test variable. The analyses were performed in the general population and, then, separately by sex. Every analysis was two tailed. A P value less than 0.05 was regarded as statistically significant. Statistical analyses were performed through the SPSS 21.0 statistical software package (IBM Corporation, Armonk, NY, USA).

\section{Results}

At the baseline, the population sample appeared to be mainly made up of middle-aged subjects, with a slightly tendency to overweight and hyperglycemia (Table 1).

Considering the whole population sample, at the univariate analysis SUA positively correlated with age, BMI, waist circumference, SBP, DBP, TC, TG, LDL-C, Apo B-100, FPG, AST and ALT, while it was inversely associated with HDL-C, Apo A-1 and eGFR (Table 2). These results were robust in the subgroup analysis by sex (Table 2).

Dividing the sample according to SUA mean (whole population $=4.8 \mathrm{mg} / \mathrm{dL}$, men $=5.6 \mathrm{mg} / \mathrm{dL}$, women $=4.1 \mathrm{mg} / \mathrm{dL}$ ), MetS appeared to be more frequent for higher concentrations of SUA $[\mathrm{OR}=2.12$, $95 \%$ C.I. $(1.55,2.90)$ in men and $\mathrm{OR}=2.69,95 \%$ C.I. $(1.91,3.78)$ in women]. In the overall population, this tendency was confirmed when only the most severe forms of MetS were considered (with 4 to 5 risk factors) $[\mathrm{OR}=2.59$, 95\%C.I. $(1.58,4.24)]$.

By drawing the ROC curves, SUA appeared a predictive test of MetS in the whole population [AUC $=0.647$, $95 \%$ C.I. $(0.609,0.686), \mathrm{P}<0.001]$ and by sex [men: $\mathrm{AUC}=0.592,95 \%$ C.I. $(0.529,654), \mathrm{P}=0.004$; women: $\mathrm{AUC}=0.758,95 \%$ C.I. $(0.711,0.806), \mathrm{P}<0.001$ ] (Fig. 2), with sex-related differences in the best cut-off values ( $5.5 \mathrm{mg} / \mathrm{dL}$ for men; $4.2 \mathrm{mg} / \mathrm{dL}$ for women). Considering only the most severe forms of MetS (4 to 5 risk factors), this finding wasconfirmed in the overall population, with an AUC of 0.643, 95\%C.I.(0.581, 0.705), P $<0.001$ and a best cut-off point of $5.1 \mathrm{mg} / \mathrm{dL}$.

Repeating prospectively the same analysis, SUA appeared again predictive of middle-term (4-year) MetS incidence in whole population [AUC $=0.604,95 \%$ C.I. $(0.518,0.690), \mathrm{P}=0.029$; with $4.7 \mathrm{mg} / \mathrm{dL}$ best cut-off value] and in the female group [AUC $=0641,95 \%$ C.I. $(0.519,0.762), \mathrm{P}=0.039$; with $3.9 \mathrm{mg} / \mathrm{dL}$ best cut-off value], but not in the male one $(\mathrm{P}>0.05)$.

\section{Discussion}

Our findings largely agree with the available literature obtained in adult subjects ${ }^{17,18}$, which suggests that hyperuricemic subjects tend to develop MetS more frequently. SUA cut-off values currently considered in the gout treatment are higher than those proposed for the cardiovascular disease prevention ${ }^{19}$. Remarkably, our findings show that SUA levels associated with preclinical CVDs risk (as MetS is) are even lower.

Oxidative stress, induced by high levels of uric acid through the inhibition of endothelial NO bioavailability ${ }^{20}$, is supposed to result in a reduction of the endothelium-mediated vasodilatation, leading to a decrease of the blood flow in insulin target tissues. At skeletal muscle level, this lowers the insulin sensitivity, leading to hyperinsulinemia and insulin resistance on the long term. In adipose tissue, uric acid induces the expression of pro-inflammatory cytokines associated with insulin resistance ${ }^{21}$ and negatively modulates the activity of the nuclear receptor PPAR- $\gamma$, which acts as insulin sensitizer ${ }^{22}$. Furthermore, at the systemic levels, the induced 


\begin{tabular}{|c|c|c|c|c|c|c|}
\hline \multirow[b]{2}{*}{ Parameters } & \multicolumn{2}{|c|}{ Whole population } & \multicolumn{2}{|l|}{ Male } & \multicolumn{2}{|l|}{ Female } \\
\hline & $\mathbf{r}$ & $\mathbf{P}$ & $\mathbf{r}$ & $\mathbf{P}$ & $\mathbf{r}$ & $\mathbf{P}$ \\
\hline Age (years) & 0.218 & $<0.001$ & 0.132 & 0.006 & 0.368 & $<0.001$ \\
\hline Body mass index $\left(\mathrm{Kg} / \mathrm{m}^{2}\right)$ & 0.353 & $<0.001$ & 0.323 & $<0.001$ & 0.387 & $<0.001$ \\
\hline Waist Circumference $(\mathrm{cm})$ & 0.500 & $<0.001$ & 0.365 & $<0.001$ & 0.468 & $<0.001$ \\
\hline Systolic blood pressure $(\mathrm{mmHg})$ & 0.237 & $<0.001$ & 0.133 & 0.006 & 0.256 & $<0.001$ \\
\hline Diastolic blood pressure (mmHg) & 0.196 & $<0.001$ & 0.101 & 0.036 & 0.094 & 0.038 \\
\hline Total cholesterol (mg/dL) & 0.161 & $<0.001$ & 0.167 & $<0.001$ & 0.214 & $<0.001$ \\
\hline Triglycerides $(\mathrm{mg} / \mathrm{dL})$ & 0.333 & $<0.001$ & 0.358 & $<0.001$ & 0.278 & $<0.001$ \\
\hline High-density lipoprotein cholesterol (mg/dL) & -0.301 & $<0.001$ & -0.217 & $<0.001$ & -0.174 & $<0.001$ \\
\hline Low-density lipoprotein cholesterol (mg/dL) & 0.157 & $<0.001$ & 0.129 & 0.007 & 0.201 & $<0.001$ \\
\hline Apolipoprotein A1 (mg/dL) & -0.312 & $<0.001$ & -0.166 & 0.001 & -0.186 & $<0.001$ \\
\hline Apolipoprotein B (mg/dL) & 0.231 & $<0.001$ & 0.141 & 0.003 & 0.256 & $<0.001$ \\
\hline Fasting plasma glucose $(\mathrm{mg} / \mathrm{dL})$ & 0.345 & $<0.001$ & 0.130 & $<0.001$ & 0.343 & $<0.001$ \\
\hline Aspartate aminotransferase (U/L) & 0.224 & $<0.001$ & 0.173 & $<0.001$ & 0.168 & $<0.001$ \\
\hline Alanine aminotransferase (U/L) & 0.209 & $<0.001$ & 0.085 & NS & 0.085 & NS \\
\hline eGFR $\left(\mathrm{mL} / \mathrm{min} / 1.73 \mathrm{~m}^{2}\right)$ & -0.286 & $<0.001$ & -0.275 & $<0.001$ & -0.467 & $<0.001$ \\
\hline
\end{tabular}

Table 2. Significant predictors of SUA in the whole population sample and by sex. eGFR, estimated glomerular filtration rate; NS, non significant.

\section{OVERALL}

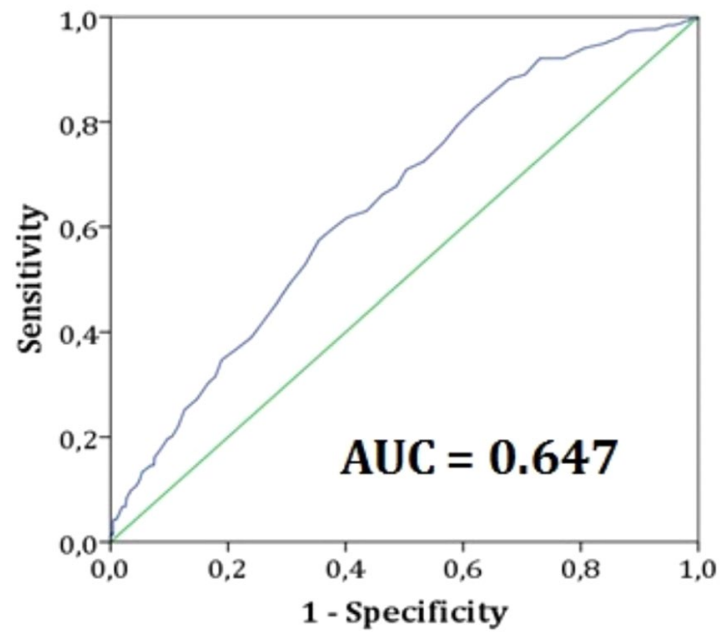

Figure 2. Receiver Operating Characteristic curve (ROC curve) constructed using serum uric acid concentrations $(\mathrm{mg} / \mathrm{dl})$ as test variable and metabolic syndrome as state variable in the general population. Data refer to the 2008's population survey.

endothelial dysfunction results in increasing the peripheral resistances, which also contribute to smooth muscle cell proliferation in the media ${ }^{23}$.

As negative modulator of the renal function, SUA may accelerate the progression of the hypertension, especially in the later stages of the natural history of hypertensive diseas $\mathrm{e}^{24}$. In this regard, several studies attested that a reduction in SUA levels with allopurinol treatment improves blood pressure values, validating his potential role in the pathogenesis of chronic hypertension ${ }^{25}$.

Numerous experimental evidence in humans and animal models, such as knockout mice for xanthine oxidoreductase $^{22}$, support also that elevated levels of uric acid are also involved in the pathogenesis of obesity ${ }^{26}$, which is another diagnostic criteria for MetS. In fact, inducing the oxidative stress through NADPH oxidase activation, SUA inhibits the synthesis of adiponectin and generates oxidized lipids and inflammatory mediators such as monocyte chemoattractant protein-1 (MCP-1), determining a gain of weight ${ }^{27}$. Moreover, the visceral fat accumulation may also cause atherogenic dyslipidemia and increase hepatic SUA production. Actually, low SUA concentrations were just proved to improve the population's heath status in general, also leading important positive economic effects ${ }^{28}$.

Thus, our findings strengthen for their part the Gerald Reaven's hypothesis, who just included SUA in MetS definition decades ago $^{29}$. 
Certainly, the present study has some limitations. Primarily, the tight inclusion criteria to the analysis has reduced the number of eligible subjects. On the other hand, this has also cleaned the population sample from strong confounding factors. Then, the administered food-frequency questionnairecould not provide valuable data about the intake of dietary purines. However, the population dietary pattern is very well-known and believed to be stable because of the previous nutritional intervention ${ }^{13}$. Finally, we were not able to identify the hypoexcretors and the hyperproducers of uric acid. However,we excluded subjects with severely compromised renal function and results were adjusted for renal impairment, such as uric acid hyperproduction is supposed to underlie the metabolic adverse effects we observed.Then, the application of some exclusion criteria could have introduced a selection bias, but the included subjects remained representative of the general older Brisighella Heart Study cohort, being age and gender matched. By the way, our results are in total agreement with what observed in the unselected older cohort of the Progetto Veneto Anziani (Pro.V.A) study ${ }^{30}$, enrolled in an area relatively near to Brisighella. Finally, the accuracy of incidence data was obviously affected by the relatively brief period offollow-up. On the other hand, a number of confounding variables could have affectedfindings over a longerperiod.

In conclusion, relying on our data, hyperuricemia appears to be a highly prevalent component of MetS, especially in the most severe forms, as well as a risk factor for MetS developing, with cut-off values far lower than those considered dangerous nowadays in older subjects, and in particular in women.

\section{References}

1. Borghi, C. \& Cicero, A. F. Serum uric acid and acute coronary syndrome: Is there a role for functional markers of residual cardiovascular risk? Int. J. Cardiol. 250, 62-63 (2018).

2. Kawasaki, M. Tissue Characterization of Coronary Plaques as a Key to Relationship between Serum Uric Acid Level and Cardiovascular Disease: A Direct Risk or an Indirect Marker? J. Atheroscler. Thromb. 23, 905-7 (2016).

3. Verdecchia, P. et al. Relation between serum uric acid and risk of cardiovascular disease in essential hypertension. The PIUMA study. Hypertension. 36, 1072-8 (2000).

4. Lee, J., Sparrow, D., Vokonas, P. S., Landsberg, L. \& Weiss, S. T. Uric acid and coronary heart disease risk: evidence for a role of uric acid in the obesity-insulin resistance syndrome. The Normative Aging Study. Am. J. Epidemiol. 142, 288-94 (1995).

5. Cicero, A. F. et al. Brisighella Heart Study Group. Serum uric acid change and modification of blood pressure and fasting plasma glucose in an overall healthy population sample: data from the Brisighella heart study. Ann. Med. 49, 275-282 (2017).

6. Cicero, A. F. et al. Brisighella Heart Study Group. High serum uric acid is associated to poorly controlled blood pressure and higher arterial stiffness in hypertensive subjects. Eur. J. Intern. Med. 37, 38-42 (2017).

7. Palmer, T. M. et al. Association of plasma uric acid with ischaemic heart disease and blood pressure: mendelian randomisation analysis of two large cohorts. BMJ. 347, f4262 (2013).

8. Borghi, C. \& Cicero, A. F. G. Serum Uric Acid and Cardiometabolic Disease: Another Brick in the Wall? Hypertension. 69, 1011-1013 (2017).

9. Kanbay, M. et al. Uric acid in metabolic syndrome: From an innocent bystander to a central player. Eur. J. Intern. Med. 29, 3-8 (2016).

10. Gersch, C. et al. Inactivation of nitric oxide by uric acid. Nucleosides Nucleotides Nucleic Acids. 27, 967-78 (2008).

11. Vincent, M. A., Barrett, E. J., Lindner, J. R., Clark, M. G. \& Rattigan, S. Inhibiting NOS blocks microvascular recruitment and blunts muscle glucose uptake in response to insulin. Am. J. Physiol. Endocrinol. Metab. 285, E123-9 (2003).

12. Abbasian, M., Ebrahimi, H., Delvarianzadeh, M., Norouzi, P. \& Fazli, M. Association between serum uric acid (SUA) levels and metabolic syndrome (MetS) components in personnel of Shahroud University of Medical Sciences. Diabetes Metab. Syndr. 10, 132-6 (2016).

13. Cicero, A. F. G., Dormi, A., D’Addato, S. \& Borghi, C. Brisighella Heart Study Staff. From risk factor assessment to cardiovascular disease risk and mortality modification: the first 40 years of the Brisighella Heart Study. Clin. Lipidol. 6, 269-276 (2011).

14. Cicero, A. F. et al. LDL-oxidation, serum uric acid, kidney function and pulse-wave velocity: Data from the Brisighella Heart Study cohort. Int. J. Cardiol. 261, 204-208 (2018).

15. Ruscica, M. et al. Brisighella Heart Study Group. Circulating Levels of Proprotein Convertase Subtilisin/Kexin Type 9 and Arterial Stiffness in a Large Population Sample: Data From the Brisighella Heart Study. J. Am. Heart. Assoc. 6, e005764, https://doi. org/10.1161/JAHA.117.005764 (2017).

16. Alberti, K. G., Zimmet, P. \& Shaw, J. Metabolic syndrome-a new world-wide definition. A Consensus Statement from the International Diabetes Federation. Diabet. Med. 23, 469-80 (2006).

17. Woo, J., Swaminathan, R., Cockram, C., Lau, E. \& Chan, A. Association between serum uric acid and some cardiovascular risk factors in a Chinese population. Postgrad. Med. J. 70, 486-91 (1994).

18. Laws, A. \& Reaven, G. M. Evidence for an indipendent relationship between insuline resistance and fasting plasma HDL. Cholesterol, triglyceride and insulin concentration. J Intern Med. 231, 25-30 (1992).

19. Richette, P. et al. 2016 updated EULAR evidence-based recommendations for the management of gout. Ann. Rheum. Dis. 76, 29-42 (2017).

20. Wong, C. K. et al. The effects of hyperuricaemia on flow-mediated and nitroglycerin-mediated dilatation in high-risk patients. Nutr. Metab. Cardiovasc. Dis. 24, 1012-9 (2014).

21. Sautin, Y. Y., Nakagawa, T., Zharikov, S. \& Johnson, R. J. Adverse effects on the classical antioxidanturic acid in adipocytes: NADPH oxidase-mediated oxidative/nitrosative stress. Am. J. Physiol. Cell Physiol. 293, C584-128 (2007).

22. Cheung, K. J. et al. Xanthine oxidoreductase is a regulator of adipogenesis and PPAR-gamma activity. Cell Metab. 5, 115-128 (2007).

23. Corry, D. B. et al. Uric acid stimulates vascular smooth muscle cell proliferation and oxidative stress via the vascular reninangiotensin system. J. Hypertens. 26, 269-75 (2008).

24. Hwu, C. M. \& Lin, K. H. Uric acid and the development of hypertension. Med. Sci. Monit. 16, RA224-30 (2010).

25. Feig, I., Soletsky, B. \& Johnson, R. J. Effect of Allopurinol on blood pressure of adolescents with Newly diagnosed essential hypertension: a randomized trial. JAMA. 300, 924-932 (2008).

26. Johnson, R. J. et al. Sugar, uric acid, and the etiology of diabetes and obesity. Diabetes. 62, 3307-15 (2013).

27. Wen, C. P. et al. Is high serum uric acid a risk marker or a target for treatment? Examination of its independent effect in a large cohort with low cardiovascular risk. Am. J. Kidney. Dis. 56, 273-288 (2010).

28. Degli Esposti, L. et al. Hyperuricemia is associated with increased hospitalization risk and healthcare costs: Evidence from an administrative database in Italy. Nutr. Metab. Cardiovasc. Dis. 26, 951-61 (2016).

29. Reaven, G. Metabolic syndrome: pathophysiology and implications for management of cardiovascular disease. Circulation. 106, 286-8 (2002)

30. Zurlo, A. et al. High serum uric acid levels increase the risk of metabolic syndrome in elderly women: The PRO.V.A study. Nutr. Metab. Cardiovasc. Dis. 26, 27-35 (2016). 


\section{Acknowledgements}

All authors acknowledge the Faenza Public Health District and all the General Practitioners of Brisighella for their continuous support to the study. This work was supported by the University of Bologna and the "Fondazione del Monte" (Bank foundation) [Grant 2016].

\section{Author Contributions}

A.F.G.C. designed the study, performed the statistical analysis and wrote the article; F.F. performed the statistical analysis and wrote the article; M.G. and E.G. performed the laboratory analysis; all of the authors collected data and critically revised the manuscript; C.B. coordinated the research group.

\section{Additional Information}

Competing Interests: The authors declare no competing interests.

Publisher's note: Springer Nature remains neutral with regard to jurisdictional claims in published maps and institutional affiliations.

(c) (1) Open Access This article is licensed under a Creative Commons Attribution 4.0 International License, which permits use, sharing, adaptation, distribution and reproduction in any medium or format, as long as you give appropriate credit to the original author(s) and the source, provide a link to the Creative Commons license, and indicate if changes were made. The images or other third party material in this article are included in the article's Creative Commons license, unless indicated otherwise in a credit line to the material. If material is not included in the article's Creative Commons license and your intended use is not permitted by statutory regulation or exceeds the permitted use, you will need to obtain permission directly from the copyright holder. To view a copy of this license, visit http://creativecommons.org/licenses/by/4.0/.

(C) The Author(s) 2018 